\title{
Physics with Tagged Forward Protons at RHIC ${ }^{\S}$
}

\author{
Kin Yip ${ }^{*}$ for the STAR Collaboration
}

Brookhaven National Lab., Collider-Accelerator Department, Upton, NY 11973, USA

\begin{abstract}
Here we describe diffractive measurements at RHIC in proton+proton collisions with a special optics run of $\beta^{*} \sim 21 \mathrm{~m}$ at STAR, at the center-of-mass energy $\sqrt{s}=200 \mathrm{GeV}$ during the the RHIC $2009 \mathrm{run}$. We present published results of single spin asymmetry as well as preliminary results on double spin asymmetries and central exclusive production.
\end{abstract}

PACS Number(s): 13.85.Dz and 13.88.+e.

Keywords: Elastic scattering, spin asymmetry.

\section{INTRODUCTION AND THEORETICAL FORMALISM}

Elastic scattering of polarized protons at small four momentum transfer squared $-t$ is described by interference of Coulomb and nuclear amplitudes. Coulomb amplitude is calculable by QED and such interference provides a unique opportunity to study the dynamics of the strong interaction in the nonperturbative region. The total cross section was measured to very high energy and turned out to be in a good agreement with the description by the Regge pole exchange. At ultra relativistic energies the main contribution comes from Pomeron or, in modern terms, multigluon exchange [1]. Most of the previous experiments were done with unpolarized beams and targets. The first measurement with polarized protons at high energies in the Coulomb nuclear interference (CNI) region $(\sqrt{s}=19.4 \mathrm{GeV})$ was done in E704 experiment [2] with moderate precision. RHIC with its polarized beams [3] published a number of accurate measurements with $\sqrt{s}=6.8-21.7 \mathrm{GeV}[4,5]$ a few years ago. But only one measurement with a limited statistics exists so far in the collider energy range [6].

Elastic scattering of two identical particles with spin $\frac{1}{2}$ is described by 5 helicity amplitudes $[7,8]$. Two amplitudes $\phi_{1}(s, t)=<++|M|++>\quad$ and $\quad \phi_{3}(s, t)=<+-|M|+->$ produce no spin-flip, two other $\phi_{2}(s, t)=<++|M|->>$ and $\phi_{4}(s, t)=<+-|M|-+>$ produce double spin-flip and the last $\phi_{5}(s, t)=<++|M|+->$ produces single spin-flip. Each

*Address correspondence to this author at the Brookhaven National Lab., Collider-Accelerator Department, Upton, NY 11973, USA;

Tel: 1-631-344-4116; Fax: 1-631-344-5954;

E-mail: kinyip@bnl.gov

${ }^{\S}$ Presented at the Low x workshop, May 30 - June 4 2013, Rehovot and Eilat, Israel. of the amplitudes can be written as a sum of hadronic and Coulomb amplitudes $\phi_{i}=\phi_{i}^{e m}+\phi_{i}^{h}$. Electromagnetic part is calculable from QED. It is believed that the main hadronic contribution to the cross section comes from non-flipping amplitudes so the optical theorem could be written as $\sigma_{\text {tot }}=\left.\frac{4 \pi}{s} \operatorname{Im}\left(\phi_{1}+\phi_{3}\right)\right|_{t=0}$. Other hadron amplitudes are expected to be small and are parametrized in terms of $\operatorname{Im} \phi_{+}=\operatorname{Im}\left(\phi_{1}+\phi_{3}\right) / 2$ :

$\varphi_{2}=2 r_{2} \operatorname{Im} \varphi_{+} \quad \varphi_{4}=\frac{-t}{m^{2}} r_{4} \operatorname{Im} \varphi_{+} \quad \varphi_{5}=\frac{\sqrt{-t}}{m} r_{5} \operatorname{Im} \varphi_{+}$

The differential cross section and asymmetries can be written in terms of the amplitudes:

$\frac{d \sigma}{d t}=\frac{2 \pi}{s^{2}}\left(\left|\varphi_{1}\right|^{2}+\left|\varphi_{1}\right|^{2}+\left|\varphi_{2}\right|^{2}+\left|\varphi_{3}\right|^{2}+\left|\varphi_{4}\right|^{2}+4\left|\varphi_{5}\right|^{2}\right)$

$A_{N} \frac{d \sigma}{d t}=-\frac{4 \pi}{s^{2}} \operatorname{Im}\left\{\varphi_{5}^{*}\left(\varphi_{1}+\varphi_{2}+\varphi_{3}-\varphi_{4}\right)\right\}$

$A_{N N} \frac{d \sigma}{d t}=\frac{4 \pi}{s^{2}}\left\{2\left|\varphi_{5}\right|^{2}+\operatorname{Re}\left(\varphi_{1}^{*} \varphi_{2}-\varphi_{3}^{*} \varphi_{4}\right)\right\}$,

$A_{S S} \frac{d \sigma}{d t}=\frac{4 \pi}{s^{2}}\left\{\operatorname{Re}\left(\varphi_{1} \varphi_{2}^{*}+\varphi_{3} \varphi_{4}^{*}\right)\right\}$

where $A_{N}$ is the single spin asymmetry and $A_{N N}$ and $A_{S S}$ are the double spin asymmetries.

\section{EXPERIMENT}

The layout of the experiment is shown in Fig. (1). Protons scattered at very small angles at the interaction point (IP) travel inside the beam pipe until they reach the Roman Pot (RP) detectors located in the RHIC tunnel on both sides of the STAR detector. Each RP contains four silicon microstrip detectors and a trigger scintillation counter. During the 2009 run, we were able to insert RP detectors to be as close as about $12 \sigma$ ( $\sigma$ being the beam size) or 10 $\mathrm{mm}$ from the center of the beam pipe. Two RP's with 
detectors inserted horizontally (at $55.5 \mathrm{~m}$ from IP) and another two RP's vertically (at $58.5 \mathrm{~m}$ ) were used at each side of IP. More details of the detectors can be found in [9]. The coordinates measured by the detectors relate to the scattering angles at IP by the transport matrix:

$\left(\begin{array}{c}x \\ y\end{array}\right)_{R P}=T_{R P} \cdot\left(\begin{array}{c}\theta_{x} \\ \theta_{y}\end{array}\right)_{I P}$,

where index ${ }_{R P}$ denotes a particular Roman Pot. The $\beta^{*}$ at STAR during our special run was about $21 \mathrm{~m}$ and the RP's were positioned to give us a parallel-to-point focusing optics. As a result, the error introduced by unknown position of the interaction point was minimal. More details on the detector layout, alignment and performance can be found at [10].

\section{ANALYSIS}

Elastically triggered events were selected for reconstructions and the cuts are briefly described below.

(A) Clusters of consecutive strips with charge values above $5 \sigma$ from their pedestals were found. We ignore rare clusters larger then 5 strips, because there were a lot of noise among them.

(B) A threshold depending on the cluster width was applied to the total charge of each cluster. This gave us better signal to noise ratio for clusters of 3 and 4 strips. After these cuts we had individual plane efficiencies above $99 \%$.

(C) Clusters in the planes of the same orientation (horizontal/x or vertical/y) within the same RP were merged and we required that their coordinates were within $200 \mu m$ (2 strips) from each other.

(D) Clusters in $\mathrm{x}$ and $\mathrm{y}$ orientations form a track and opposite pairs of tracks formed from each side of the IP were chosen.

(E) Transport equation (5) was solved for each side.

(F) The strongest criteria of elastic events selection is the collinearity cut which was realized by requiring $\chi^{2}$, where $\quad \chi^{2}=\left(\theta_{x}^{\text {west }}-\theta_{x}^{\text {east }}\right)^{2} / \sigma_{x}^{2}+\left(\theta_{y}^{\text {west }}-\theta_{y}^{\text {east }}\right)^{2} / \sigma_{y}^{2}$ and $\sigma_{x}$ and $\sigma_{y}$ are typically $\approx 58 \mu \mathrm{rad}$, to be $<9$. The correlation between the angles can be seen in Fig. (2).

About 21 millions events out of about 33 million elastic triggers written during the run were selected for asymmetry calculations.

Using the square root formula $[6,11]$, raw asymmetry as function of azimuthal angle $\phi$ for only ++ and -- bunch polarizations can be written as:

$$
\begin{aligned}
& \varepsilon_{N}(\varphi)=\frac{\left(P_{B}+P_{Y}\right) A_{N} \cos (\varphi)}{1+\delta(\varphi)}= \\
& \frac{\sqrt{N^{++}(\varphi) N^{--}(\pi-\varphi)}-\sqrt{N^{--}(\varphi) N^{++}(\pi-\varphi)}}{\sqrt{N^{++}(\varphi) N^{--}(\pi-\varphi)}+\sqrt{N^{--}(\varphi) N^{++}(\pi-\varphi)}}
\end{aligned}
$$

where $\delta(\phi)=P_{B} P_{Y}\left(A_{N N} \cos ^{2}(\phi)+A_{S S} \sin ^{2}(\phi)\right), N^{i j}(\phi)-$ number of events with bunch polarization pattern $i j$ at the azimuthal angle $\phi . P_{B / Y}$ are polarizations of the blue and yellow beams, measured by HJET and pCarbon polarimeters [12]. The polarization values averaged for the time of our
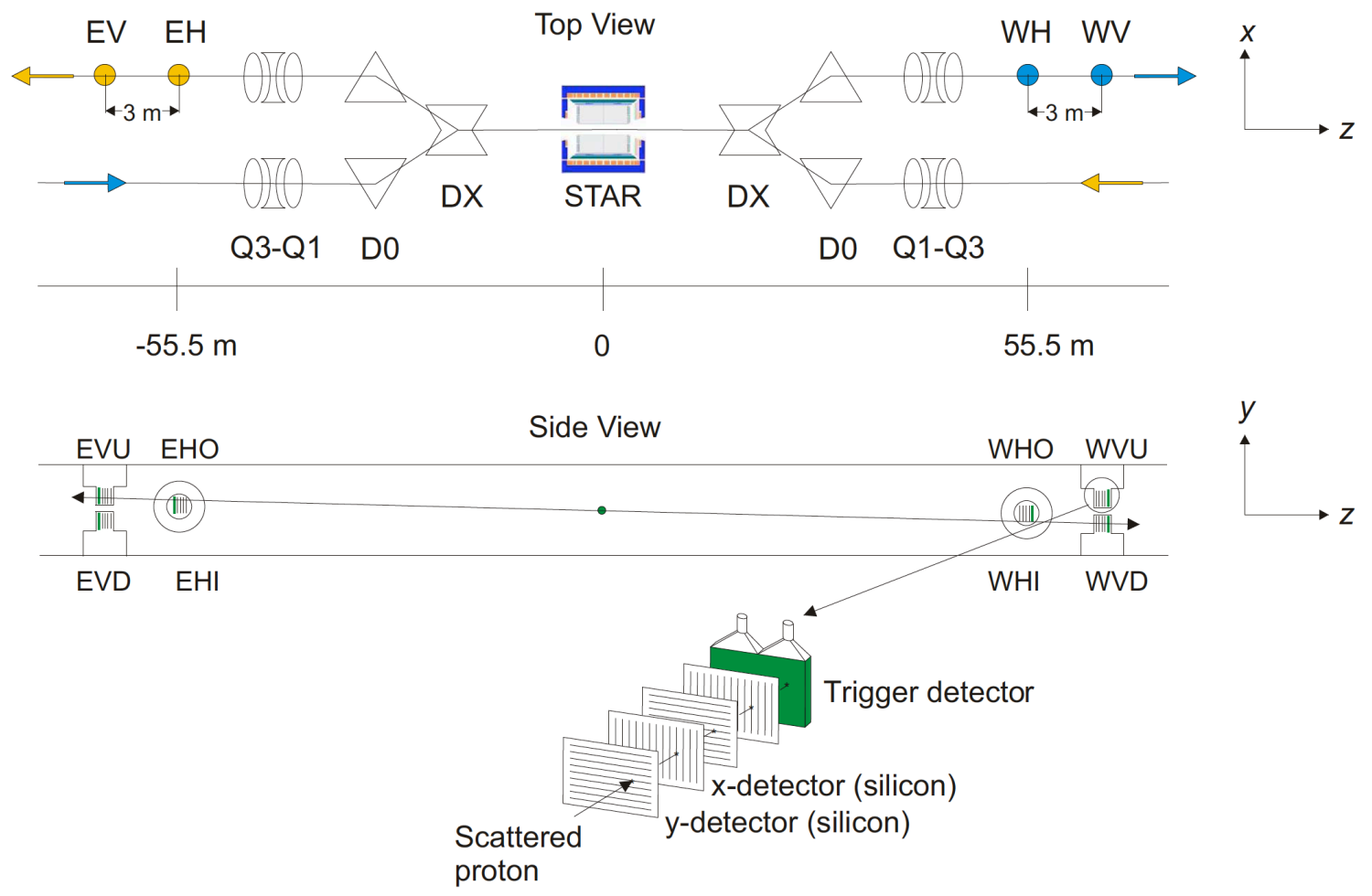

Fig. (1). Layout of the setup for small-t measurements with the STAR detector (in the center). 

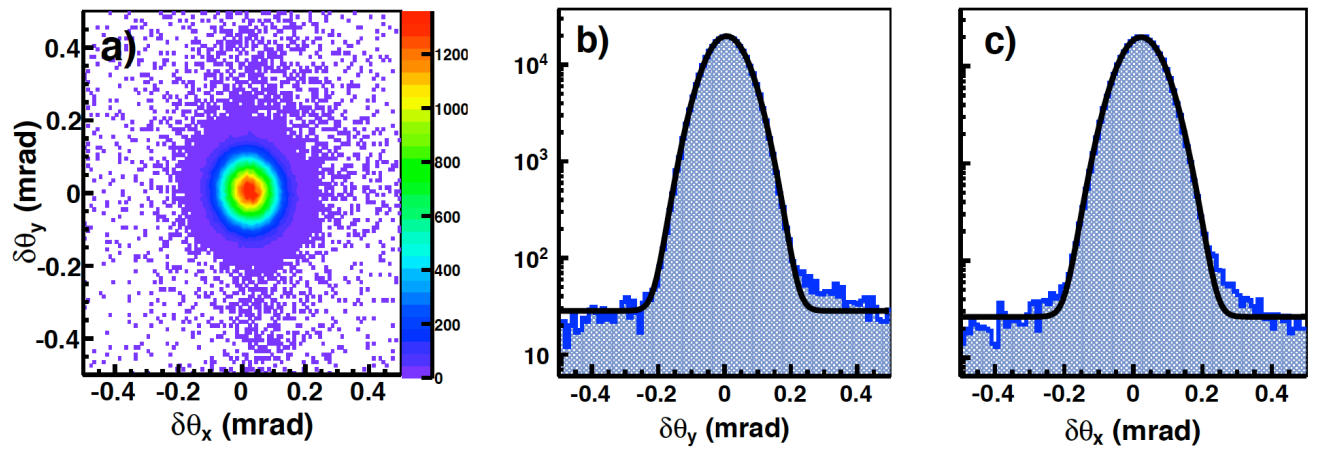

Fig. (2). (color online) Distribution of $\delta \theta_{y} v s \delta \theta_{x}$ for both detector pairs in horizontal RPs (a) and their projections in $\delta \theta_{y}$ (b) and $\delta \theta_{x}$ (c). The overlaid curves represent the fits with a Gaussian signal and a linear background. The $\sigma$ values of distributions are $\approx 58 \mu$ rad, consistent with beam angular divergence, and the background-to-signal ratio under the Gaussian distributions in $\pm 3 \sigma$ is $\approx 0.4 \%$.

data taking were: $P_{B}+P_{Y}=1.224 \pm 0.066, P_{B}-P_{Y}=-0.016 \pm 0.066$ and $P_{B} P_{Y}=0.375 \pm 0.041$ (errors shown here include global systematic uncertainties). From double spin asymmetries measured by [6], we know that $\delta(\phi)$ is less than 0.01 . Using other different bunch polarization combinations, other raw asymmetries can be introduced similarly to (6); particularly, the so-called "wrong combination" is shown here:

$\varepsilon_{N}^{\prime}(\varphi)=\frac{\left(P_{B}+P_{Y}\right) A_{N} \cos (\varphi)}{1-\delta(\varphi)}=$

$\frac{\sqrt{N^{+-}(\varphi) N^{-+}(\pi-\varphi)}-\sqrt{N^{-+}(\varphi) N^{+-}(\pi-\varphi)}}{\sqrt{N^{+-}(\varphi) N^{-+}(\pi-\varphi)}+\sqrt{N^{-+}(\varphi) N^{+-}(\pi-\varphi)}}$,
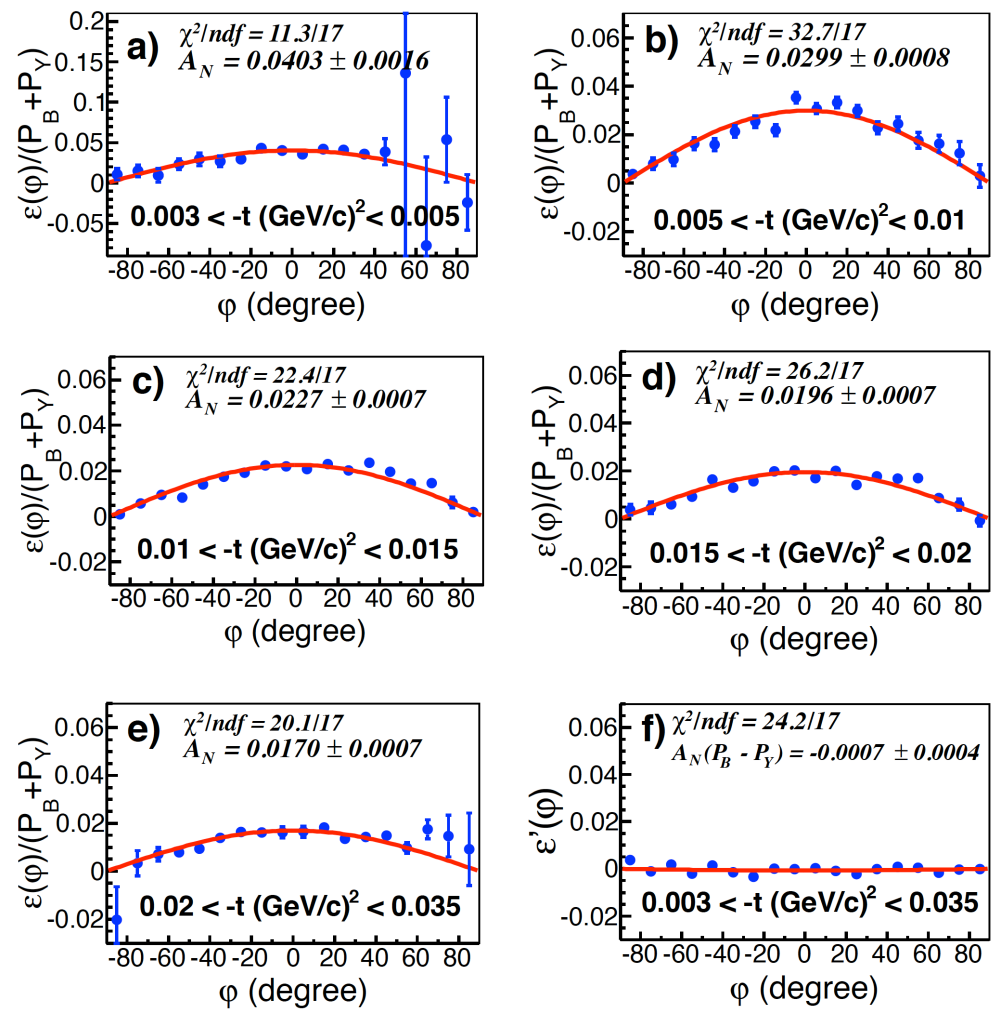

the whole $t$-range are presented in Fig. (3). Using (6), we fitted the raw asymmetry to extract $A_{N}$ 's in $5 t$-bins.

Double spin raw asymmetries $A_{N N}$ and $A_{S S}$ can be extracted from the following equation (as there is no square root formula available):

$$
\begin{aligned}
& \delta(\varphi)=P_{B} P_{Y}\left(A_{N N} \cos ^{2}(\varphi)+A_{S S} \sin ^{2}(\varphi)\right)= \\
& \frac{\left(\frac{N^{++}}{L^{++}}+\frac{N^{--}}{L^{--}}\right)-\left(\frac{N^{+-}}{L^{+-}}+\frac{N^{-+}}{L^{-+}}\right)}{\left(\frac{N^{++}}{L^{++}}+\frac{N^{--}}{L^{--}}\right)+\left(\frac{N^{+-}}{L^{+-}}+\frac{N^{-+}}{L^{-+}}\right)}
\end{aligned}
$$


Here $L^{i j}$ are relative luminosity monitors for the corresponding polarization pattern.

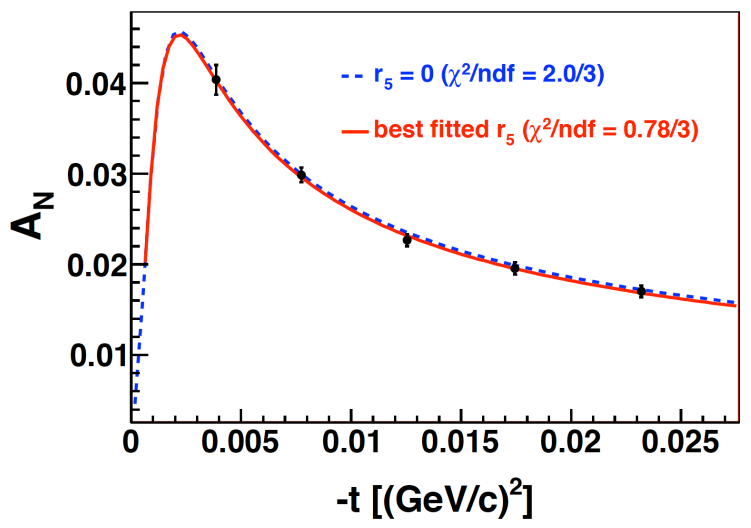

Fig. (4). (color online) The measured single spin asymmetry $A_{N}$ for five $-t$ intervals. Vertical error bars show statistical uncertainties. Statistical error bars in $-t$ are smaller than the plot symbols. The dashed curve corresponds to theoretical calculations without hadronic spin-flip and the solid one represents the $r_{5}$ fit.

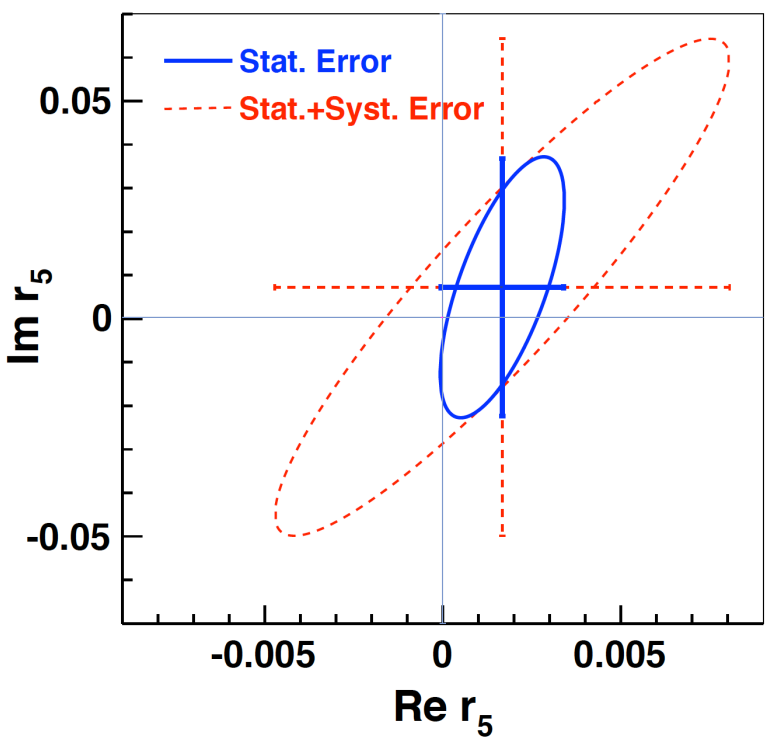

Fig. (5). (color online) Fitted value of $r_{5}$ with contours corresponding to statistical error only (solid ellipse and cross) and statistical+systematic errors (dashed ellipse and cross) of $1 \sigma$.

\section{RESULTS ON ELASTIC SCATTERING MEASUREMENTS}

The recently published results [13] on the single spin asymmetry are shown in Fig. (4) in comparison with theoretical curve without hadron spin-flip and with the best fit allowing non-zero hadronic spin-flip (see [14] for formula). Only statistical uncertainties have been included. The value of $r_{5}$ resulting from the fit described above is shown in Fig. (5), together with both statistical and systematic uncertainties. The obtained values $\operatorname{Re} r_{5}=$ $0.0017 \pm 0.0063$ and $\operatorname{Im} r_{5}=0.007 \pm 0.057$ are consistent

with the hypothesis of no hadronic spin-flip contribution $\left(\phi_{5}\right.$ ) at the energy of this experiment. That is, only Pomeron exchange, which contributes only to spin non-flipping amplitudes $\phi_{1}$ and $\phi_{3}$, seems to survive at high energies.

The preliminary results on double spin raw asymmetries are shown in Fig. (6). Though some effects of the order of $10^{-3}$ could be seen, they are small. Here we have used relative luminosities obtained from counts of inelastic triggers produced by the vertex position detector and beambeam counters (BBC). However, after more thorough studies, BBC coincidence counts were proved to be the least sensitive to double spin effects with negligible statistical uncertainty. The systematic error due to the normalization uncertainty of BBC coincidence counts on $\delta\left(\frac{A_{N N}+A_{S S}}{2}\right)$ is at the level of $8.4 \times 10^{-4}$.

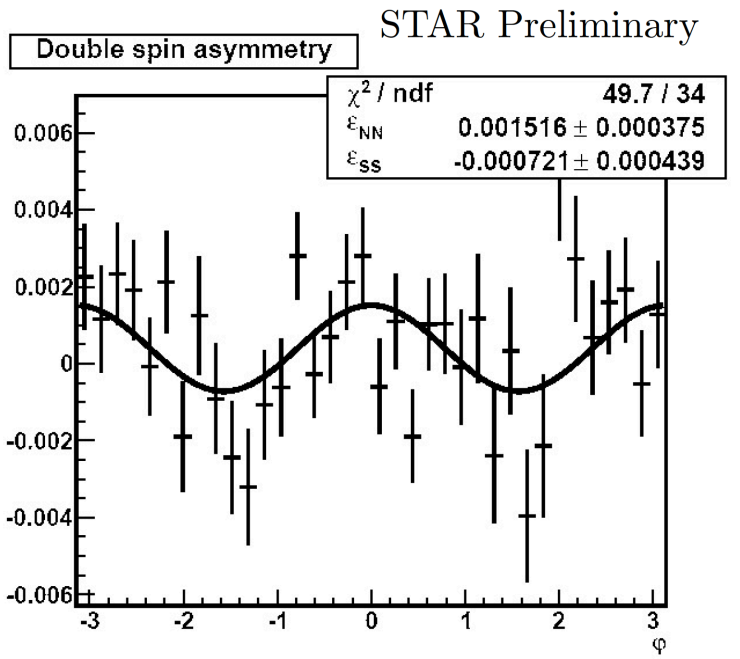

Fig. (6). STAR Preliminary results on double spin raw asymmetry for the entire t-interval.

\section{CENTRAL EXCLUSIVE PRODUCTION}

We have also studied the invariant mass spectrum of the two oppositely charged pions produced in the Ce ntral Exclusive Production process of $p p \rightarrow p \pi^{+} \pi^{-} p$. Our ability to tag protons in the Roman Pots on opposite sides of the IP helps reduce background in this measurement of double Pomeron exchange compared to standard hadronic production processes. Pions were obtained from tracks with $\mathrm{dE} / \mathrm{dx}$ identification. It is also required that the protons be noncollinear with scattering angle separation more than 0.15 mrad to avoid cosmics. Moreover, the missing transverse momentum of the final states two pions and two protons) had to be less than $0.02 \mathrm{GeV}$.

In Fig. (7), the spectrum of the invariant mass of $\pi^{+} \pi^{-}$ pairs produced with the above cuts is presented. The likesign background is very small, which gives a measure of exclusiveness of the process. The spectrum is not corrected for acceptance, but preliminary acceptance study indicates that corrections would not change shape of the spectrum significantly. Presented spectrum is similar to the one published by the AFS Collaboration at ISR [15] as it shows the same characteristic features : 
- $\quad$ it is dominated by low invariant mass pairs, $m_{\pi \pi}$, below $1 \mathrm{GeV}$;

- $\quad$ it shows the same characteristic drop around $1 \mathrm{GeV}$ which may be due to $\pi^{+} \pi^{-}$rescattering or $f_{0}(980)$ interference with the S-wave background [16].

\section{Invariant mass $\pi \pi$}

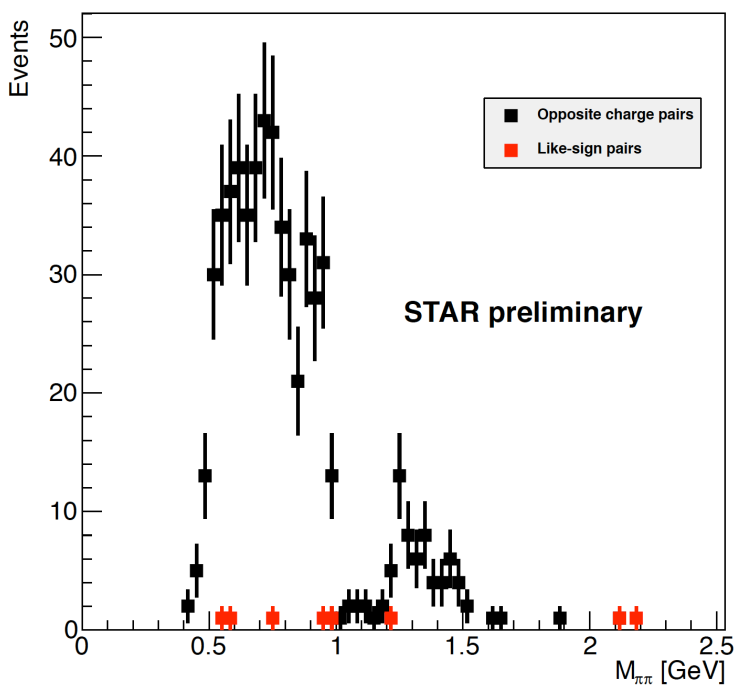

Fig. (7). (color online) STAR Preliminary results on invariant mass spectrum of $\pi^{+} \pi^{-}$pairs produced in the central exclusive process $p+p \rightarrow p+\pi^{+} \pi^{-}+p$ at $\sqrt{s}=200 \mathrm{GeV}$.

\section{SUMMARY AND FUTURE PROSPECTS}

We had a very successful run with the physics program with tagged forward protons at RHIC in 2009, in which over 70 million events (including 33 million events with elastic triggers) were collected. We have published our results on single spin asymmetry $\left(A_{N}\right.$ and $\left.r_{5}\right)$ and we are finalizing our results on double spin asymmetries as well as the central exclusive production. In the mean time, we are also preparing for the Phase II of this physics program, in which we change our detector configurations so that we will not need a special optics of RHIC and thus this physics program can be carried out simultaneously with other physics programs of the STAR experiment. This will allow us to take much more data and explore other physics possibilities at RHIC.

\section{CONFLICT OF INTEREST}

The author confirms that this article content has no conflicts of interest.

\section{ACKNOWLEDGEMENTS}

We thank the RHIC Operations Group and RCF at BNL, the NERSC Center at LBNL, the KISTI Center in Korea and the Open Science Grid consortium for providing resources and support. This work was supported in part by the Offices of NP and HEP within the U.S. DOE Office of Science, the U.S. NSF, CNRS/IN2P3, FAPESP CNPq of Brazil, Ministry of Ed. and Sci. of the Russian Federation, NNSFC, CAS, MoST and MoE of China, the Korean Research Foundation, GA and MSMT of the Czech Republic, FIAS of Germany, DAE, DST, and CSIR of India, National Science Centre of Poland, National Research Foundation (NRF-2012004024), Ministry of Sci., Ed. and Sports of the Rep. of Croatia, and RosAtom of Russia.

\section{REFERENCES}

[1] (a). Nussinov S. Colored-Quark Version of Some Hadronic Puzzles. Phys Rev Lett 1975; 34: 1286-1289. (b). Nussinov S. Perturbative recipe for quark-gluon theories and some of its applications. Phys Rev 1976; D14: 246-57.

[2] Akchurin N, Langland J, Onel Y, et al. Analyzing power measurement of $p p$ elastic scattering in the Coulomb-nuclear interference region with the $200-\mathrm{GeV} / c$ polarized-proton beam at Fermilab. Phys Rev 1993; D48: 3026-36.

[3] Bai M, Roser T, Ahrens L, et al. Polarized proton collisions at $205 \mathrm{GeV}$ at RHIC. Phys Rev Lett 2006; 96: 174801-1-4.

[4] (a). Okada H, Alekseev IG, Bravar A, et al. Measurement of the analyzing power $\mathrm{A}_{\mathrm{N}}$ in $p p$ elastic scattering in the CNI region with a polarized atomic hydrogen gas jet target. Phys Lett 2005; B638: 450-4. (b). Alekseev IG, Bravar A, Bunce G, et al. Measurements of single and double spin asymmetry in $p p$ elastic scattering in the CNI region with a polarized atomic hydrogen gas jet target. Phys Rev 2009; D79: 094014$1-18$.

[5] Bazilevsky A. Measurements of the energy dependence of the analyzing power in $p p$ elastic scattering in the CNI region. J Phys Conf Ser 2011; 295: 012096-1-5.

[6] (a). Bültmann $\mathrm{S}$, Chiang IH, Chrien RE, et al. First measurement of $\mathrm{A}_{\mathrm{N}}$ at $\sqrt{s}=200 \mathrm{GeV}$ in polarized proton-proton elastic scattering at RHIC. Phys Lett 2006; B632: 167-72. (b). Bültmann S, Chiang IH, Chrien RE, et al. Double spin asymmetries $A_{\mathrm{NN}}$ and $A_{\mathrm{SS}}$ at $\sqrt{s}=200 \mathrm{GeV}$ in polarized proton-proton elastic scattering at RHIC. Phys Lett 2007; B647: 98-103.

[7] Buttimore NH, Gotsman E, Leader E. Spin-dependent phenomena induced by electromagnetic-hadronic interference at high energies. Phys Rev 1978; D18: 694-716.

[8] Trueman TL. Hadronic spin dependence and the use of coulombnuclear interference as a polarimeter. 1996 [arXiv:hep-ph/9610316].

[9] Bültmann S, Chen W, Chiang IH, et al. The PP2PP experiment at RHIC: silicon detectors installed in Roman Pots for forward proton detection close to the beam. Nucl Instr Meth 2004; A535: 415-20.

[10] Plyku D. Performance of silicon detectors in polarized proton-proton elastic scattering at RHIC. J Phys Conf Ser 2011; 295: 012129-1-5.

[11] Ohlsen GG, Keaton PW Jr. Techniques for measurement of spin- $1 / 2$ and spin-1 polarization analyzing tensors. Nucl Instr Meth 1973; 109: 4159.

[12] http://www4.rcf.bnl.gov/ cnipol/pubdocs/Run09Offline/

[13] Adamczyk L, Agakishiev G, Aggarwal MM, et al. [STAR Collaboration]. Single spin asymmetry $\mathrm{A}_{\mathrm{N}}$ in polarized proton-proton elastic scattering at $\sqrt{s}=200 \mathrm{GeV}$. Phys Lett 2013; B719: 62-9.

[14] Buttimore NH, Kopeliovich BZ, Leader E, Soffer J, Trueman TL. Spin dependence of high energy proton scattering. Phys Rev 1999; D59: 114010-1-18.

[15] Akesson T, Albrow MG, Almehed S, et al. [Axial Field Spectrometer Collaboration]. A search for glue balls and a study of double pomeron exchange at the CERN intersecting storage rings. Nucl Phys 1986; B264: 154-84.

[16] Dedonder J-P, Furman A, Kaminski R, Lesniak L, Loiseau B. S-, Pand D-wave final state interactions and CP violation in $\mathrm{B}^{ \pm} \rightarrow \pi^{ \pm} \pi^{\mp} \pi^{ \pm}$ decays. Acta Phys Polon 2011; B42: 2013-43. 